Journal of

Cancer Research and Therapeutic Oncology

\title{
Retrospective Analysis of 5-Year Survival Rate of Nasopharyngeal Carcinoma: Correlation with Clinical Features and Prognosis
}

\author{
Clement Arthur ${ }^{1}$, Hao Ruan ${ }^{1}$, Xiaofeng Wang ${ }^{1}$, Xue Jun Zhou ${ }^{1}$, Sha Liu ${ }^{2}$, Ping Zhou ${ }^{2}$ Collins Koranteng Osei ${ }^{1}$, \\ Joseph Akparibilla Azure ${ }^{3}$, Xun $\mathrm{Bi}^{4}$, ZhongLin $\mathrm{Mu}^{1 \star}$
}

${ }^{1}$ Department of Otorhinolaryngology Head and Neck Surgery, Hainan Medical University, First Affiliated Hospital Haikou, 570102, Hainan Province, China

${ }^{2}$ Department of Radiotherapy, Hainan Medical University, First Affiliated Hospital Haikou, 570102, Hainan Province, China

${ }^{3}$ Department of Obstetrics and Gynecology, Hainan Medical University, First Affiliated Hospital, Haikou, 570102, Hainan

Province, China

${ }^{4}$ Department of General Surgery, Hainan Medical University, First Affiliated Hospital Haikou, 570102, Hainan Province, China

*Corresponding author: ZhongLin Mu, Department of Otolaryngology Head and Neck Surgery, Hainan Medical University, First Affiliated Hospital, Haikou, 570102, Hainan Province, China; Tel: +86-898-68893601; E-mail: muzhonglin2@sina.com

Received Date: January 07, 2019; Accepted Date: March 04, 2019; Published Date: March 06, 2019

Citation: Clement Arthur, et al. (2019) Retrospective Analysis of 5-Year Survival Rate of Nasopharyngeal Carcinoma: Correlation with Clinical Features and Prognosis. J Cancer Res Therap Oncol 7: 1-16.

\begin{abstract}
Main Objective: This study was designed to evaluate the epidemiological characteristics of NPC and their prognostic value, with a goal to correlate with Clinical Features and Prognosis.

Results: The 5-year overall survival (OS) rates for patients in stages I, II, III and IV were $66.7 \%, 55.6 \%, 41.8 \%$ and $25.9 \%$, respectively $(\mathrm{P}=0.026)$, while the respective 5 -year progression-free survival (PFS) rates were $60.0 \%, 51.1 \%, 36.6 \%$ and $18.6 \%$ $(\mathrm{P}=0.044)$. Clinical staging appears to be the most important prognostic factor for NPC. As the stage number increases, both the 5-year OS and PFS significantly decrease.

The respective 5-year OS rates, according to stage, for the group that received radiotherapy combined with chemotherapy (Concurrent Chemotherapy-CCRT or Neo-adjuvant Chemotherapy-NAC-) and for the group that received radiotherapy only were as follows: stages I and II, $62.7 \%$ and 58.7\% (P=0.450); stage III, 34.0\%, 28.6\% ( $\mathrm{P}=0.460)$; stage IV, 36.7\%, and $0.0 \%(\mathrm{P}=0.036)$. The respective 5-year PFS rates in these groups were as follows: stages I and II, $56.0 \%$ and $50.2 \%(\mathrm{P}=0.550)$; stage III, $26.9 \%$ and $15.0 \%(\mathrm{P}=0.025)$; stage IV, $23.6 \%$ and $0.0 \%(\mathrm{P}=0.008)$. Patients with stages I or II NPC will likely not benefit from the addition of chemotherapy, in terms of long-term survival and PFS. However, for patients with stage III NPC, adding chemotherapy can improve PFS to a certain degree though it may not improve OS and in patients with stage IV NPC, the addition of chemotherapy can significantly prolong both OS and PFS.
\end{abstract}

Keywords: Retrospective analysis; nasopharyngeal carcinoma (NPC); Treatment; Prognosis

C2019 The Authors. Published by the JScholar under the terms of the Creative Commons Attribution License http://creativecommons.org/licenses/by/3.0/, which permits unrestricted use, provided the original author and source are credited. 


\section{Introduction}

NPC is a much more common malignancy in Southeast Asia, especially in the southern coastal area of Mainland China and in Hong Kong, Macao, and Taiwan. The annual incidence rate among the male population in Hong Kong is about 20/100,000 [1]. The incidence of NPC gradually increases with age, peaking at 50-59 years of age, and then tends to decrease [1]. Furthermore, the prognosis for those with NPC tends to worsen with age [1]. There is a notable difference in the pathological types of NPC that occur within different regions. The keratinizing type of NPC (WHO Type I) mainly occurs in Western countries with overall low incidences of NPC. However, NPC tends to be of an undifferentiated, nonkeratinizing subtype (WHO Type III) in South China and in Southeast Asian countries where the overall incidence of NPC is higher [1]. Patient survival rates also differ depending on the different pathological type of NPC. Patients with Type III have significantly better survival rates compared to those with Type I $[2,3]$.

Surgical resection is very difficult due to the fact that NPC is anatomically deep and occurs close to important neurovascular structures. Thus, the mainstay strategies for the treatment of NPC are radiotherapy-based, comprehensive therapies, including concurrent chemoradiotherapy, as well as induction or adjuvant chemotherapy and palliative chemotherapy following radiotherapy [4]. Prognosis is affected by treatment approach, race, histological type, and disease stage $[3,5,6]$ Many factors that impact the long-term survival of NPC patients have not yet been fully clarified [79]. In the current study, the clinical data from NPC patients in the Hainan Province was retrospectively analyzed in order to clarify epidemiological characteristics, influencing factors and patient survival.

The main purpose of the study was to know the overall five-year survival rate of Nasopharyngeal Carcinoma cases. It is significance study; this would indicate all the favorable and poor prognostic factors which determine the OS and PFS for NPC.

The aim of the study was achieved during the analysis of the below factors; through the indicators used and their prognosis. The study reveals that clinical stage is the most important factor for NPC. As the stage number increases, both the 5-year OS and PFS significantly decrease. The study further reveals females have favorable prognostic factors; gender is a major factor in the incidence of NPC and slightly indicative an independent prognostic factor affecting OS and PFS. In the current study, the five-year survival rate in patients older than 50 years of age was significantly lower than in patients younger than 50 years of age. Less than 50 -year is an advantage prognostic factor. The addition of chemotherapy to radiotherapy improves the outcome of the treatment.

\section{Patients and Methods}

\section{Ethical Consideration Issues}

Prior to conducting the study approval of the head of ethics committee, Head of Research Management Institute, Head of Department Otorhinolaryngology Head and Neck, Head of Department Radiotherapy, Head of Department Oncology, Head of Department Medical Records was sought before activities carried out. Upon applying through the research department then further to the ethical consideration committee, the committee, however, verified the purposes and significance of the study. Additionally, the committee verified each researcher information thoroughly and their capability to participate and conduct the study. The ethics consideration committee further organized a meeting with the researchers and the importance of the study was explained to the committee and their full consent sought and approved.

\section{Data collection}

Clinical data was collected from all histologically confirmed, new cases of 183 NPC, which occurred between 2007 and 2011, including 135 males and 48 females, aged 1285 year old, with an average age as $(47.94 \pm 13.250)$ years at Hainan Medical College First Affiliated Hospital. Patients that had been previously diagnosed with NPC and treated, but who had relapsed during this period, were excluded. However, the study did include patients that had been diagnosed with NPC outside of First Affiliated Hospital between 2007 and 2011, who then underwent subsequent treatment and follow-up at Hainan Medical College First Affiliated Hospital.

Data collected included patient demographics, NPC stage, histological type, treatment modalities, treatment efficacy, and survival time. Patient demographic characteristics included gender, age, and marital status. Age data were divided into two groups: those younger than 50 years of age and those 50 years of age and older. The disease was restaged in accordance with the eighth edition of the American Joint Committee of Cancer (AJCC) TNM staging system [10] and 
the tumor pathological types were determined according to the WHO's NPC classification [11].

\section{Radiotherapy Modalities}

The treatment modalities were radiotherapy alone, neoadjuvant chemotherapy followed by radiotherapy, concurrent chemoradiotherapy plus adjuvant chemotherapy, or palliative treatment. The agents used in neo-adjuvant chemotherapy were cisplatin combined with 5-FU. The concurrent chemoradiotherapy and adjuvant chemotherapy treatments were the same as those used by Zhang et al. [12]. Palliative treatment of advanced tumors included single-agent chemotherapy or combined chemotherapy and radiotherapy.

All patients in the three groups underwent radiotherapy with Cobalt 60 gamma photons using conventional techniques. Two laterally opposed fields were used to treat the nasopharynx and the upper neck and an anterior cervical field with a midline shield were used to treat the lower neck to 38.4-40 Gy. Thermoformable mask was used for contention. The remainder dose of irradiation was delivered via an anterior nasal field which included the nasopharynx and a large anterior cervical field treating all cervical nodes.

External beam radiotherapy was delivered with two modalities. One hundred and twenty-seven patients were treated with conventional fractionation and 56 patients with hyperfractionated radiotherapy $(1.6 \mathrm{~Gy} \times 2$ /day with an interval of 6 hours, 5 days per week). The hyperfractionated schedule was used in a previous randomized phase III trial [13]. The total dose to the nasopharynx and involved neck areas was 70-75Gy and was 50-55Gy to the remaining cervical areas from level II to level V.

\section{Examinations and Follow-up}

Patients were examined prior to treatment and during the follow-up period after treatment. Examinations included a complete medical history and physical examination, a craniofacial examination (including dental and cranial nerve exams), nasopharyngo-fiberscope, a complete blood count, serum biochemistry, a chest X-ray, and a CT or MRI examination of the nasopharynx, skull base and any suspicious metastatic sites, including the paranasal sinuses. Treatment efficacy was evaluated using the WHO criteria [14]. After treatment, the patients were asked to return to the clinic once every three months, for two years and then every six months until relapse or death. The follow-up period was defined as the period from the date of diagnosis until death or until the last follow-up time. Patients with disease recurrence, progression, and those that were lost to follow-up were considered to have died on the day of their last follow-up. Local recurrence was confirmed by examination of the nasopharynx, head, and neck and was verified by needle aspiration biopsy or MRI. Distant metastases were identified by clinical symptoms, physical examination, or bone scans and verified by CT or MRI scan(s).

\section{Assessment and Statistical Analysis}

Prognostic factors for NPC patients were determined by analyzing the associations between patient survival and the following: age, gender, disease-stage, NPC histological type, treatment modalities, and primary therapeutic effects. Overall survival (OS) was defined as the time from diagnosis to the time of death from any cause. The cut-off time for patients who survived was defined as the time of the last visit. Progression-free survival (PFS) referred to the time from the start of treatment until recurrence, disease progression or death from any cause. The cut-off time for the cases without disease progression was defined as the time of the last visit. SPSS 19.0 (SPSS Inc, Chicago, IL, USA) was used for statistical analysis. The survival curves were analyzed using the Kaplan-Meier method and the survival curves of different groups of patients were compared using a log-rank test. The Cox proportional hazard model was used for multivariate analysis of the prognostic factors, including the patient, tumor and treatment modalities. A total of 183 newly diagnosed cases of NPC were treated in Hainan Medical College First Affiliated Hospital between January 1, 2007, and December 31, 2011. The age of disease onset was similar to the normal distribution, with a median age of 48.0 years (Table 1).

Patients were followed every 3 months during the first 2 years, every 6 months for the next 3 years, and then every year until death). The 5 -year survival rate for all patients that underwent follow-up was $49.8 \%$, however, the median survival (5-year) was not reached and could not be calculated. The 5-year survival rates for patients with stages I, II, III, and IV NPC were $66.7 \%, 55.6 \%, 41.8 \%$, and $25.9 \%$, respectively. There was a significant difference in the survival curves among patients in different clinical stages $(\mathrm{P}=0.026)$ (Figure1). The prognostic significances of age, gender, $\mathrm{T}$ stage, $\mathrm{M}$ stage, primary treatment modality, and therapeutic effect were evaluated by univariate analysis (Table 2 ). Favorable prognostic 


\begin{tabular}{|c|c|c|}
\hline Characteristics & Number & $\%$ \\
\hline Age(year) & $47.94 \pm 13.250$ & \\
\hline Range & $12-85$ & \\
\hline Median & 48.00 & \\
\hline Male: Female & $135: 48$ & \\
\hline \multicolumn{3}{|l|}{ Histology } \\
\hline WHOType I & 20 & 10.9 \\
\hline WHOType II & 41 & 22.4 \\
\hline WHOType III & 122 & 6.7 \\
\hline \multicolumn{3}{|l|}{ Stage(AJCC2010) } \\
\hline StageI & 15 & 8.2 \\
\hline StageII & 45 & 24.6 \\
\hline StageIII & 96 & 52.5 \\
\hline StageIV & 27 & 14.8 \\
\hline \multicolumn{3}{|l|}{ T Classification } \\
\hline $\mathrm{T} 1$ & 35 & 19.1 \\
\hline $\mathrm{T} 2$ & 73 & 39.9 \\
\hline $\mathrm{T} 3$ & 56 & 30.6 \\
\hline $\mathrm{T} 4$ & 19 & 10.4 \\
\hline \multicolumn{3}{|l|}{ N Classification } \\
\hline N0 & 43 & 23.5 \\
\hline N1 & 83 & 45.4 \\
\hline N2 & 48 & 26.2 \\
\hline N3 & 9 & 4.9 \\
\hline \multicolumn{3}{|l|}{ M Classification } \\
\hline M0 & 178 & 97.3 \\
\hline M1 & 5 & 2.7 \\
\hline \multicolumn{3}{|l|}{ Primary Treatment } \\
\hline RT & 20 & 11.0 \\
\hline $\mathrm{NAC}+\mathrm{RT}+\mathrm{CCRT}$ & 14 & 24.0 \\
\hline $\mathrm{CCRT}+\mathrm{RT}+\mathrm{AC}$ & 119 & 65.0 \\
\hline
\end{tabular}

Table 1: Patients Characteristics

AJCC, America Joint Committee on Cancer; RT, Radiotherapy, NAC, Neo-adjuvant Chemotherapy, CCRT, Concurrent Chemotherapy 


\begin{tabular}{|c|c|c|c|c|c|c|c|}
\hline \multicolumn{8}{|c|}{ Univariate analysis of prognostic factors for OS and PFS } \\
\hline Factors & $\mathrm{n}()$ & $\begin{array}{l}5 \text {-year OS } \\
\text { rate }(\%)\end{array}$ & $\mathrm{x} 2$ & ${ }^{*} \mathrm{P}$ value & $\begin{array}{l}\text { 5year PFS } \\
\text { rate }(\%)\end{array}$ & $\mathrm{x} 2$ & ${ }^{\star} \mathrm{P}$ value \\
\hline \multicolumn{8}{|l|}{ Age-group } \\
\hline $\begin{array}{l}<50 \\
50 \text { and above }\end{array}$ & $\begin{array}{l}103 \\
80\end{array}$ & $\begin{array}{l}58.3 \\
41.3\end{array}$ & 5.208 & 0.022 & $\begin{array}{l}53.4 \\
32.5\end{array}$ & 4.773 & 0.029 \\
\hline \multicolumn{8}{|l|}{ Gender } \\
\hline $\begin{array}{l}\text { Male } \\
\text { Female }\end{array}$ & $\begin{array}{l}135 \\
48\end{array}$ & $\begin{array}{l}43.8 \\
60.3\end{array}$ & 4.15 & 0.042 & $\begin{array}{l}39.6 \\
59.3\end{array}$ & 4.38 & 0.036 \\
\hline \multicolumn{8}{|l|}{$\begin{array}{l}\text { Stage } \quad \text { (AJCC } \\
2014)\end{array}$} \\
\hline $\begin{array}{l}\text { Stage I } \\
\text { Stage I I } \\
\text { Stage I I I } \\
\text { Stage IV }\end{array}$ & $\begin{array}{l}15 \\
45 \\
96 \\
27\end{array}$ & $\begin{array}{l}66.7 \\
55.6 \\
41.8 \\
25.9\end{array}$ & 9.275 & 0.026 & $\begin{array}{l}60.0 \\
51.1 \\
36.6 \\
18.6\end{array}$ & 8.103 & 0.044 \\
\hline \multicolumn{8}{|l|}{$\mathrm{T}$ classification } \\
\hline $\begin{array}{l}\mathrm{T} 1 \\
\mathrm{~T} 2 \\
\mathrm{~T} 3 \\
\mathrm{~T} 4\end{array}$ & $\begin{array}{l}35 \\
73 \\
56 \\
19\end{array}$ & $\begin{array}{l}57.1 \\
54.4 \\
41.4 \\
21.3\end{array}$ & 9.113 & 0.028 & $\begin{array}{l}51.4 \\
50.7 \\
35.7 \\
15.8\end{array}$ & 9.737 & 0.021 \\
\hline \multicolumn{8}{|l|}{ M classification } \\
\hline $\begin{array}{l}\text { M0 } \\
\text { M1 }\end{array}$ & $\begin{array}{l}178 \\
5\end{array}$ & $\begin{array}{l}50.6 \\
0.00\end{array}$ & 4.975 & 0.026 & $\begin{array}{l}48.3 \\
0.00\end{array}$ & 4.558 & 0.035 \\
\hline \multicolumn{8}{|l|}{ Primary treatment } \\
\hline $\begin{array}{l}\text { RT } \\
\text { NAC+RT+CCRT } \\
\text { CCRT+RT+AC }\end{array}$ & $\begin{array}{l}20 \\
44 \\
119\end{array}$ & $\begin{array}{l}42.1 \\
50.4 \\
69.7\end{array}$ & 8.921 & 0.012 & $\begin{array}{l}36.8 \\
45.5 \\
67.2\end{array}$ & 10.50 & 0.005 \\
\hline Treatment & Effects & & & & & & \\
\hline $\begin{array}{l}\text { NC } \\
\text { PD } \\
\text { PR } \\
\text { CR }\end{array}$ & $\begin{array}{l}11 \\
14 \\
34 \\
124\end{array}$ & $\begin{array}{l}9.1 \\
14.4 \\
47.1 \\
56.5\end{array}$ & 16.567 & 0.001 & $\begin{array}{l}0.0 \\
7.1 \\
41.2 \\
52.4\end{array}$ & 20.061 & 0.000 \\
\hline
\end{tabular}

Table 2: Univariate analysis of prognostic factors for OS and PFS

AJCC, America Joint Committee on Cancer; RT, Radiotherapy, NAC, Neo-adjuvant Chemotherapy; AC, Adjuvant Chemotherapy, CCRT, Concurrent Chemotherapy; CR, complete response; PR, partial response; NC, no change; PD, progression disease; OS, overall survival; PFS, progression free survival 


\begin{tabular}{|c|c|c|c|c|c|c|c|}
\hline & No. of & OS & & & & PFS & \\
\hline Variable & Patients & $\begin{array}{l}\text { Hazard } \\
\text { ratio }\end{array}$ & $95 \% \mathrm{CI}$ & ${ }^{* P}$ value & Hazard ratio & $95 \% \mathrm{CI}$ & ${ }^{*} \mathrm{P}$ value \\
\hline Age group $<50$ & 103 & 1.12 & $1.41-3.82$ & 0.043 & 2.052 & $1.20-3.05$ & 0.017 \\
\hline 50 and above & 80 & & & & & & \\
\hline \multicolumn{8}{|l|}{ Gender } \\
\hline Male & 135 & 0.35 & $0.147-0.817$ & 0.01 & 0.383 & $0.189-0.776$ & 0.07 \\
\hline Female & 48 & & & & & & \\
\hline \multicolumn{8}{|l|}{ Histology } \\
\hline WHO Type I & 20 & 2.57 & $0.841-1.853$ & 0.088 & 1.408 & 0.667-2.969 & 0.368 \\
\hline WHO Type II & 41 & & & & & & \\
\hline WHO Type III & 122 & & & & & & \\
\hline \multicolumn{8}{|l|}{ Stage (AJCC 2010) } \\
\hline Stage I & 15 & 0.748 & $0.317-1.764$ & 0.034 & 1.008 & $0.502-2.025$ & 0.029 \\
\hline Stage II & 45 & & & & & & \\
\hline Stage III & 96 & & & & & & \\
\hline Stage IV & 27 & & & & & & \\
\hline \multicolumn{8}{|l|}{ T classification } \\
\hline $\mathrm{T} 1$ & 35 & 1.427 & $0.610-3.337$ & 0.025 & 1.05 & $0.54-2.039$ & 0.000 \\
\hline $\mathrm{T} 2$ & 73 & & & & & & \\
\hline $\mathrm{T} 3$ & 56 & & & & & & \\
\hline $\mathrm{T} 4$ & 19 & & & & & & \\
\hline \multicolumn{8}{|l|}{$\mathrm{N}$ classification } \\
\hline N0 & 43 & 0.904 & $0.464-1.758$ & 0.765 & 1.232 & $0.622-2.437$ & 0.547 \\
\hline $\mathrm{N} 1$ & 83 & & & & & & \\
\hline $\mathrm{N} 2$ & 48 & & & & & & \\
\hline $\mathrm{N} 2$ & 9 & & & & & & \\
\hline \multicolumn{8}{|l|}{ M classification } \\
\hline M0 & 178 & 4.278 & $\begin{array}{l}0.679- \\
26.940\end{array}$ & 0.019 & 4.304 & $0.697-26.576$ & 0.046 \\
\hline \multicolumn{8}{|l|}{ M1 } \\
\hline \multicolumn{8}{|l|}{ Primary treatment } \\
\hline RT & 20 & 2.114 & $\begin{array}{l}1.123 \\
-3.978 \\
\end{array}$ & 0.000 & 0.636 & $0.322-1.254$ & 0.010 \\
\hline $\mathrm{NAC}+\mathrm{RT}+\mathrm{CCRT}$ & 44 & & & & & & \\
\hline $\mathrm{CCRT}+\mathrm{RT}+\mathrm{CCRT}$ & 119 & & & & & & \\
\hline \multicolumn{8}{|l|}{ Treatment Effects } \\
\hline $\mathrm{NC}$ & 14 & 1.165 & $0.611-2.224$ & 0.004 & 1.364 & $0.696-2.674$ & 0.030 \\
\hline $\mathrm{PD}$ & 17 & & & & & & \\
\hline $\mathrm{PR}$ & 34 & & & & & & \\
\hline CR & 118 & & & & & & \\
\hline
\end{tabular}

Table 3: Multivariate analysis prognostic factors for OS and PFS

AJCC, America Joint Committee on Cancer; RT, Radiotherapy, NAC, Neo-adjuvant Chemotherapy, CCRT, Concurrent Chemotherapy; CR, complete response; PR , partial response; NC, no change; PD, progression disease; OS, overall survival; PFS, progression free-survival; CI, confidence interval. 


\begin{tabular}{|c|c|c|c|c|c|c|}
\hline Factors & $\mathrm{N}=183$ & $\mathrm{RT}(\mathrm{n}=20)$ & $\mathrm{NAC}+\mathrm{RT}+\mathrm{CCRT}(\mathrm{n}=44)$ & $\mathrm{CCRT}+\mathrm{RT}+\mathrm{AC}(\mathrm{n}=119)$ & $\mathrm{x} 2$ & ${ }^{\star} \mathrm{P}$ value \\
\hline \multicolumn{7}{|l|}{ Age group } \\
\hline Less than 50 & 103 & 13 & 20 & 65 & 1.961 & 0.375 \\
\hline 50 and above & 80 & 7 & 23 & 54 & & \\
\hline \multicolumn{7}{|l|}{ Gender } \\
\hline Male & 135 & 10 & 34 & 91 & 1.201 & 0.319 \\
\hline Female & 48 & 7 & 10 & 31 & & \\
\hline \multicolumn{7}{|l|}{ Histology } \\
\hline WHO Type I & 20 & 1 & 5 & 14 & 3.374 & 0.497 \\
\hline WHO Type II & 41 & 7 & 7 & 27 & & \\
\hline WHO Type III & 122 & 12 & 32 & 78 & & \\
\hline \multicolumn{7}{|l|}{ Stage(AJCC2010) } \\
\hline Stage I & 15 & 15 & 2 & 28 & 70.487 & 0.000 \\
\hline Stage II & 45 & 3 & 24 & 30 & & \\
\hline Stage III & 96 & 1 & 2 & 47 & & \\
\hline Stage IV & 27 & 1 & 16 & 14 & & \\
\hline \multicolumn{7}{|l|}{ T classification } \\
\hline $\mathrm{T} 1$ & 35 & 15 & 2 & 28 & 49.873 & 0.000 \\
\hline $\mathrm{T} 2$ & 73 & 3 & 17 & 37 & & \\
\hline T3 & 56 & 1 & 9 & 40 & & \\
\hline $\mathrm{T} 4$ & 19 & 1 & 16 & 14 & & \\
\hline \multicolumn{7}{|l|}{ M classification } \\
\hline M0 & 178 & 20 & 44 & 119 & 8.434 & 0.004 \\
\hline M1 & 5 & & 1 & 4 & & \\
\hline
\end{tabular}

Table 4: Characteristics of patients' different primary treatment modalities

AJCC, America Joint Committee on Cancer; RT, Radiotherapy, NAC, Neo-adjuvant Chemotherapy, CCRT, Concurrent Chemotherapy; M0, no distance metastasis; M1, distance metastasis 


\begin{tabular}{|c|c|c|c|c|c|c|c|c|c|c|c|}
\hline \multirow[b]{2}{*}{$\begin{array}{l}\text { Variable No. } \\
\text { of }\end{array}$} & \multirow[b]{2}{*}{ RT } & \multirow[b]{2}{*}{$\begin{array}{l}\text { NAC } \\
+\mathrm{RT}+ \\
\text { CCRT }\end{array}$} & \multirow[b]{2}{*}{$\begin{array}{l}\text { CCRT } \\
+\mathrm{RT}+ \\
\mathrm{AC}\end{array}$} & \multicolumn{4}{|c|}{ OS rate $(\%)$} & & \multicolumn{3}{|c|}{ PFS rate (\%) } \\
\hline & & & & RT & & $\begin{array}{l}\text { NAC+ } \\
\text { RT+ } \\
\text { CCRT }\end{array}$ & & & & & $\begin{array}{l}\mathrm{C} \mathrm{CRT+} \\
\mathrm{RT}+ \\
\mathrm{AC}\end{array}$ \\
\hline Patients $=183$ & & $(n=20)$ & $(\mathrm{n}=44)$ & $(n=119)$ & $\mathrm{x} 2$ & $\begin{array}{l}{ }^{*} \mathrm{P} \\
\text { value }\end{array}$ & $(\mathrm{n}=20)$ & $(\mathrm{n}=44)$ & $(\mathrm{n}=119)$ & $\mathrm{x} 2$ & ${ }^{\star} \mathrm{P}$ value \\
\hline \multicolumn{12}{|l|}{$\begin{array}{l}\text { Stage } \\
(\text { AJCC2010) }\end{array}$} \\
\hline Stage I & 60 & 34 & 58.3 & 62.7 & 62.451 & 0.00 & 26.9 & 43.9 & 56 & 61.202 & 0.000 \\
\hline Stage II & 96 & 28.6 & 44.3 & 58.7 & & & 15.0 & 36.1 & 50.2 & & \\
\hline Stage III & 27 & 0.0 & 20.0 & 36.7 & & & 0.0 & 10.5 & 23.6 & & \\
\hline \multicolumn{12}{|l|}{$\begin{array}{l}\mathrm{T} \\
\text { classification }\end{array}$} \\
\hline $\mathrm{T} 1$ & 35 & 28.9 & 47.54 & 60.0 & 0.028 & 0.060 & 25.36 & 44.32 & 54.4 & 0.025 & 0.067 \\
\hline $\mathrm{T} 2$ & 73 & 20.9 & 34.6 & 47.3 & & & 19.6 & 26 & 45.7 & & \\
\hline T3 & 59 & 17.1 & 26.1 & 45.0 & & & 15.3 & 17.2 & 33.6 & & \\
\hline $\mathrm{T} 4$ & 19 & 5.26 & 22.9 & 44.4 & & & 4.9 & 12.3 & 30 & & \\
\hline \multicolumn{12}{|l|}{$\begin{array}{l}\mathrm{N} \\
\text { classification }\end{array}$} \\
\hline N0 & 43 & 39.2 & 44.4 & 52.1 & 0.165 & 0.059 & 30.2 & 38.3 & 47.8 & 0.122 & 0.070 \\
\hline N1 & 83 & 30.2 & 38.6 & 46.9 & & & 28.3 & 23.3 & 46.5 & & \\
\hline N2 & 48 & 14.5 & 29.5 & 44.1 & & & 10.4 & 21.6 & 22.7 & & \\
\hline $\mathrm{N} 3$ & 9 & 8.3 & 11.1 & 12.6 & & & 1.2 & 2. 0 & 7.6 & & \\
\hline \multicolumn{12}{|l|}{$\begin{array}{l}\text { M } \\
\text { classification }\end{array}$} \\
\hline M0 & 178 & 29.21 & 54.8 & 60.6 & 0.629 & 0.73 & 11.7 & 32.02 & 46.4 & 4.957 & 0.175 \\
\hline M1 & 5 & 0 & 8.87 & 10.2 & & & 0.0 & 1.53 & 5.70 & & \\
\hline
\end{tabular}

Table 5: Five -year OS and PFS rates of different stages and treatment modalities

AJCC, America Joint Committee on Cancer; RT, Radiotherapy, NAC, Neo-adjuvant Chemotherapy, CCRT, Concurrent Chemotherapy; OS, overall survival; PFS, progression free-survival; M0, no distance metastasis; M1 , distance metastasis. 
indicators for relatively longer OS included being less than 50 years of age, being female, having an earlier $\mathrm{T}$ stage, the absence of distant metastases, having had treatment with Concurrent Chemotherapy combined with radiotherapy and Neo-Adjuvant Chemotherapy, and achieving remission after first-line treatment $(\mathrm{P}<0.05)$. Age, gender, $\mathrm{T}$ stage, $\mathrm{M}$ stage, primary treatment modality, and initial therapeutic effect were all independent prognostic factors for OS, as indicated by multivariate Cox regression analysis (Table 3 ).

as $50-55 \mathrm{~Gy}$ to the remaining cervical areas from level II to level V.

The 5-year PFS rate was $42.95 \%$ for all patients. The PFS rates for patients with stages I, II, III, and IV NPC were $60.0 \%, 51.1 \%, 36.6 \%$, and $18.6 \%$, respectively. Just like the median survival, the median PFS (5-year) was not reached either. Clinical stage was found to be a significant prognostic indicator for PFS $(\mathrm{P}<0.05)$ (Figure 2). Univariate analysis showed that gender, $\mathrm{T}$ stage, $\mathrm{M}$ stage, primary treatment modality, and treatment efficacy were all prognostic factors for PFS in patients (Table 2). Favorable prognostic indicators for relatively long PFS included being a woman, having an earlier $\mathrm{T}$ stage, having no distant metastases, having had concurrent Chemotherapy combined with radiotherapy and achieving remission after primary treatment $(\mathrm{P}<0.05)$. Age, gender, $\mathrm{T}$ stage, $\mathrm{M}$ stage, the primary treatment modality, and the efficacy of first-line therapy were all independent prognostic factors for PFS, as indicated by multivariate analysis (Table 3).

The 5-year OS and PFS rates were significantly better in the combined radiotherapy (Concurrent ChemotherapyCCRT or Neo-adjuvant Chemotherapy-NAC-) groups compared with the group that received the only radiotherapy. The effect of treatment modality on long-term survival was further examined via sub-group analysis of the demographic characteristics, pathological type and stage of NPC in these two patient groups (Table 4). The group treated with a combination of chemotherapy and radiotherapy (Concurrent Chemotherapy-CCRT or Neo-adjuvant ChemotherapyNAC-) groups was again at a significantly later stage of NPC, on average, than the group that was treated with radiotherapy alone, indicating that the patients were not randomly assigned to each group and that more late-stage patients received radiotherapy combined with chemotherapy. Longterm survival was further analyzed according to different subgroups excluding interference due to unbalanced stage distribution between the three groups (Table 5). When comparing the treatment modalities, there was no significant difference in the 5-year OS and PFS of patients with stages I and II NPC ( $\mathrm{P}=0.450$ and $\mathrm{P}=0.550$, respectively) (Figure 3 ). The 5-year PFS in stage III patients treated with a combination of radiotherapy and chemotherapy was significantly better than stage III patients that were treated with radiotherapy alone $(\mathrm{P}=0.025)$, however, there was no significant difference in the 5-year OS of stage III patients when comparing these treatment modalities ( $\mathrm{P}=0.460)$ (Figure 4). The 5-year OS and PFS were both significantly better for stage IV patients that received combined chemotherapy and radiotherapy compared with stage IV patients that were treated with radiotherapy alone $(\mathrm{P}=0.036$ and $\mathrm{P}=0.008)$ (Figure 5).

\section{Results}

Gender indicates a major factor incidence of nasopharyngeal carcinoma: from the study occurrence of nasopharyngeal carcinoma, males (135) and females (48) had shown as NPC in males is $2-3$ times than in females in (Table 2, Table 3).

Age group has an impact on survival: Overall survival and Progression-free survival is worse in $>50 \mathrm{yrs}$. than $<50$ yrs. (P value 0.022 and 0.029 ). The 5 -year Overall survival and Progression-free survival for all patients were $49.8 \%$ and $42.9 \%$ and 5 -year OS rate; male and females $43.8 \%$ and $60.8 \%$ (P-value 0.042), 5-year PFS rate; male and female 39.6\% and 59.3\% (P-value 0.044) captioned as shown elsewhere and reported in (Table 2, Figure 1 and Figure 2).

The findings on 5-year Overall survival and Progression-free survival stages I, II, III, IV were $66.7 \%$, $55.6 \%, 41.8 \%$ and $25.9 \%$ and $60.0 \%, 51.1 \%, 36.6 \%$, and $18.6 \%$ observed in (Table 2, Figure $3 \mathrm{~A}$ and B) respectively.

The study indicated the results obtained according to stages and treatment modalities for overall survival and Progression-free survival as indicated below;

Overall survival for CCRT+RT+ AC stages I and II: $62.7 \%$ and $58.7 \%$, NAC +RT +CCRT stages II and III: $43.6 \%$ and $20.0 \%$, RT alone for stages III \& IV: $36.7 \%$ and $0.0 \%$. The Progression-free survival for CCRT+RT+ AC stages I and II: $56.0 \%$ and $50.2 \%$, NAC +RT +CCRT stages II and III: $36.1 \%$ and $10.1 \%$, RT alone for stages III \& IV: $23.6 \%$ and $0.0 \%$, the CR (complete remission) were 56.5\%, PR Partial remission) of $47.1 \%$ with significant 5-year OS and PFS (p-value: 0.001 and 0.000 ) captioned and shown elsewhere and reported (Table 2), Figure 3A), (Figure 4 A, B), and (Figure 5 A, B ). 


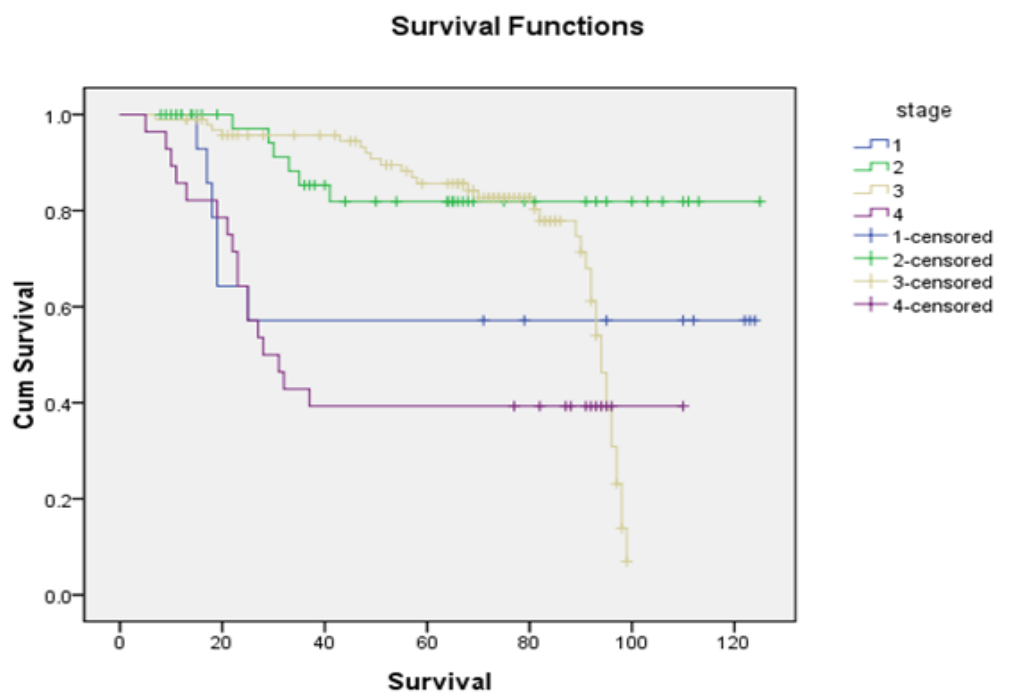

Figure: 1 Comparison of OS among AJCC stages. OS, overall survival; AJCC, American Joint Committee on Cancer

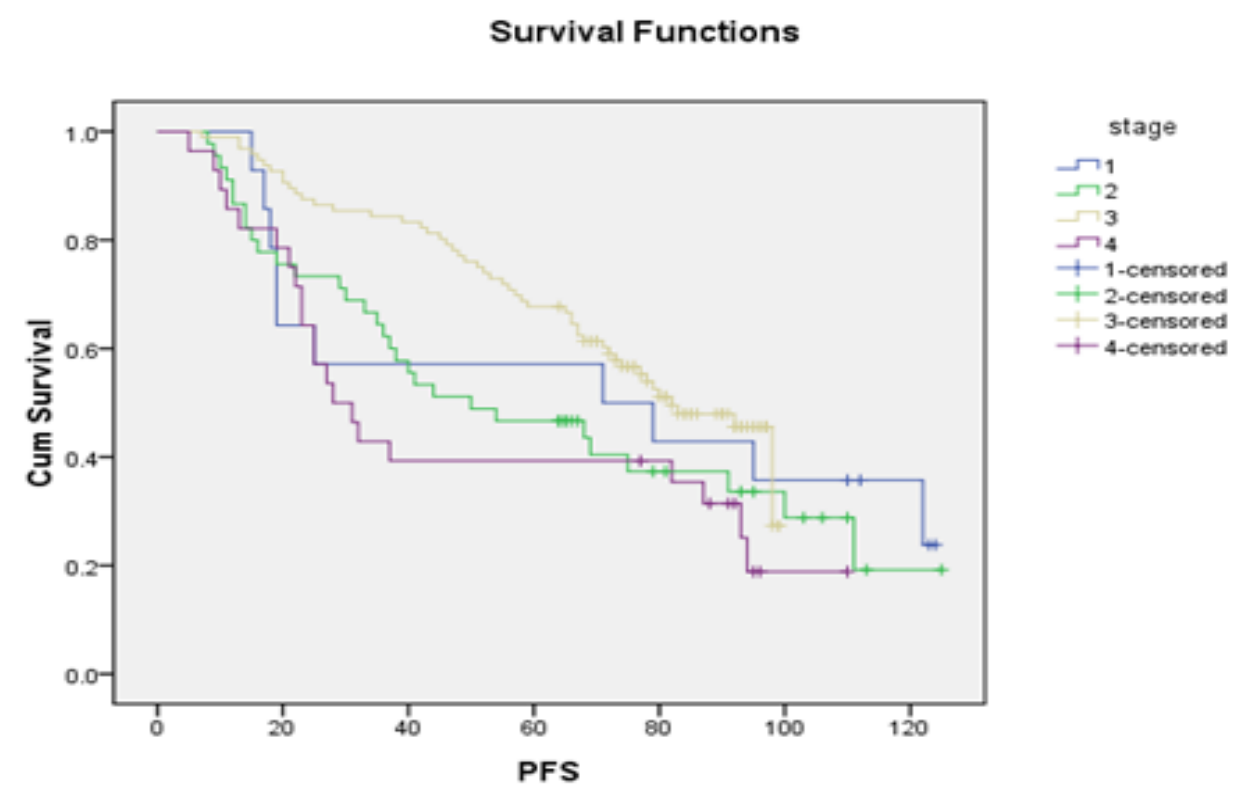

Figure: 2 Comparison of PFS among AJCC stages. PFS, progression free survival; AJCC, American Joint Committee on Cancer 

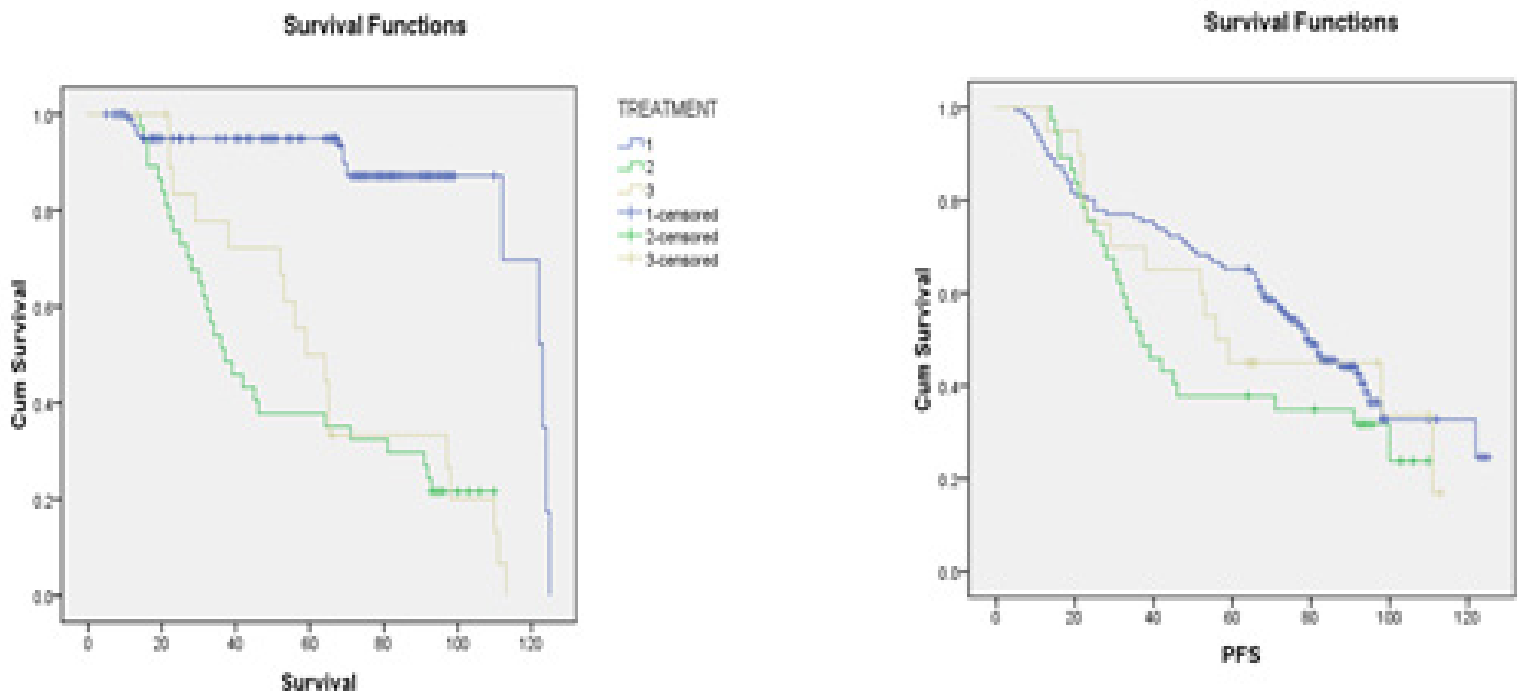

TREATMEAT

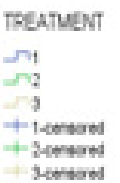

ת1

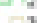

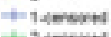

+-2terount

sertat

Figure: 3 Comparison of OS (A) and PFS (B) between the treatment methods in stage I-II. OS, overall survival; PFS progressionfree survival.
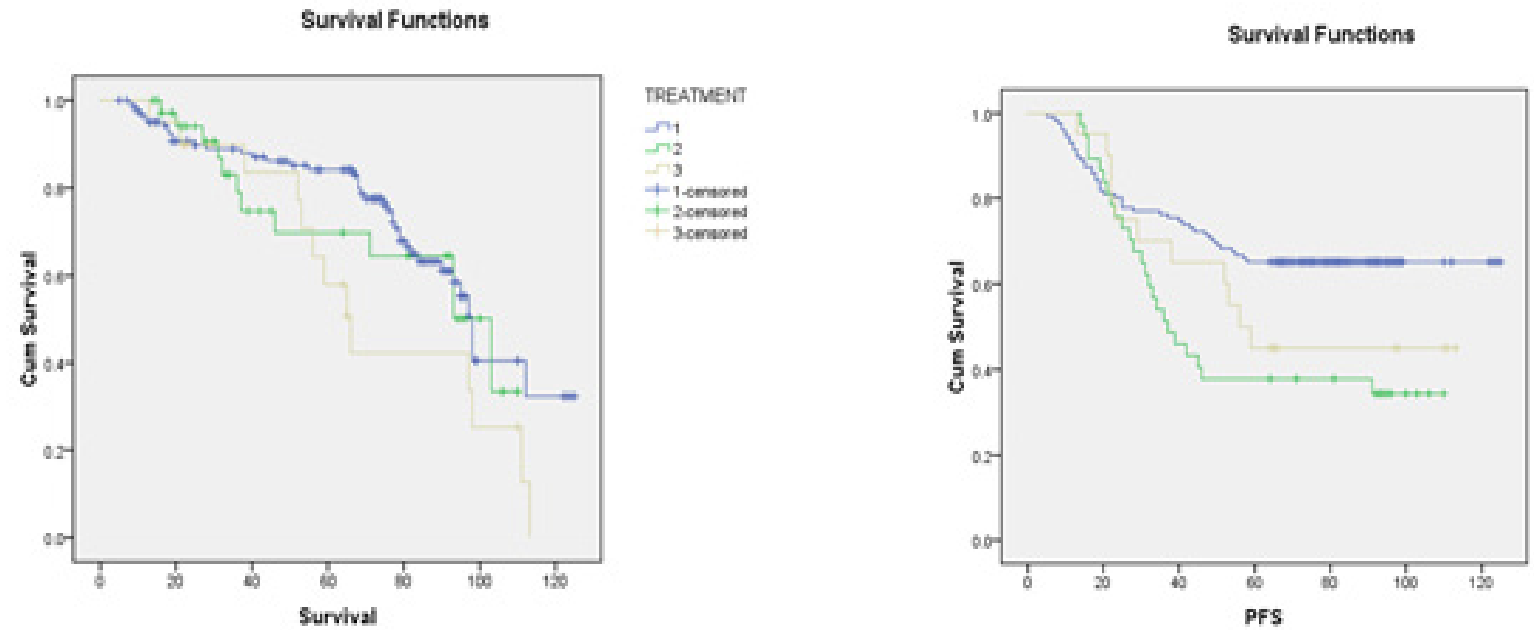

TREATMENT

TREATMEIT

${ }_{n=1}$

3

+1-sentors
+ 2cemared

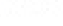

$\rightarrow$

$-\pi^{2}$

+ Eenocios

reserasos

Figure: 4 Comparison of OS (A) and PFS between the treatment methods in stage III. OS, overall survival; PFS, progression-free survival. 

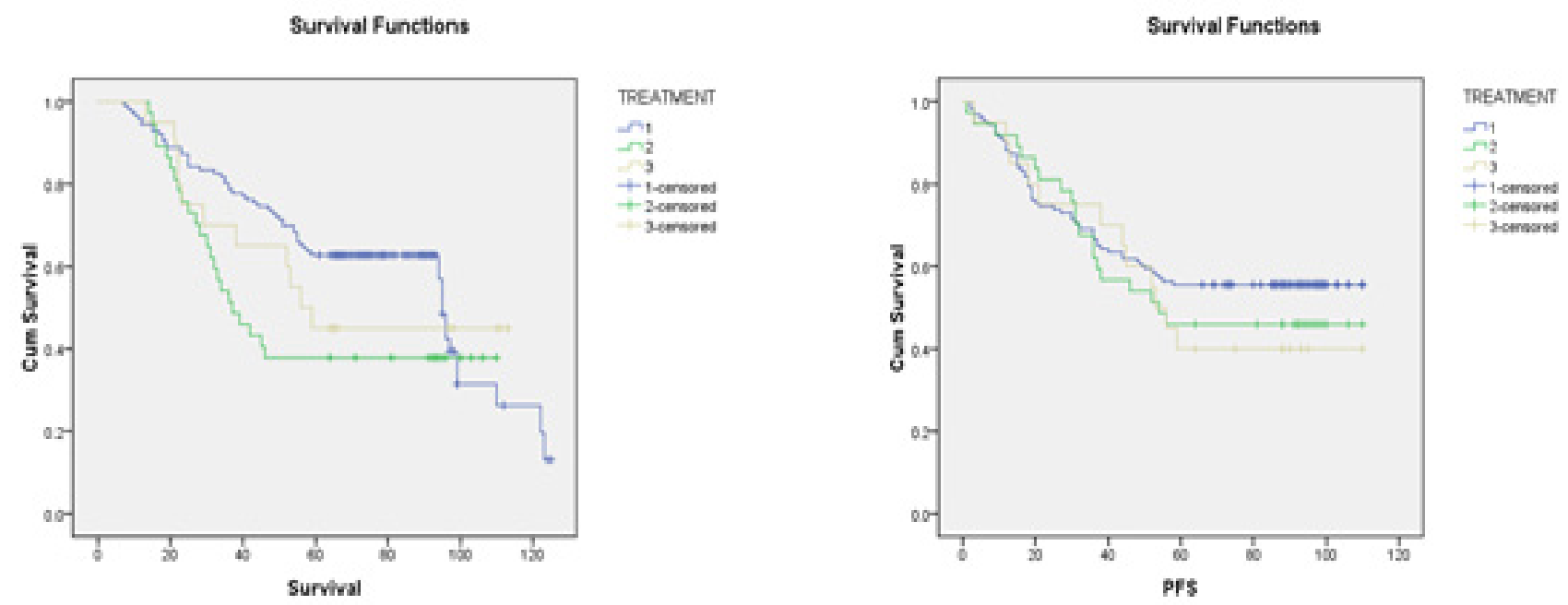

Figure 5: Comparison of OS (A) and PFS between the treatment methods in stage IV. OS, overall survival; PFS, progression-free survival.

WHO type I \& II; accounts for 33.3\%, WHO type III; recorded 6.7\%, the Overall survival ( $\mathrm{p}$-value: 0.088), ( $\mathrm{P}$ value 0.388 ) respectively. 5- Year Overall survival and Progressionfree survival rates indicate that $\mathrm{T} 1: 35$ were 2.68 and 3.25 times that of patients with T4: 19 in (Table 1, 2, 3, 4 and 5).

\section{Discussion}

In the current study, the five-year survival rate in patients older than 50 years of age was significantly lower than in patients younger than 50 years of age $(\mathrm{P}=0.022)$, similarly there was also a significant difference in 5 -year PFS between the two age groups $(\mathrm{P}=0.029)$, this indicates that the prognosis for OS in older patients is worse than in younger patients. Age was also found to be an independent prognostic factor affecting the long-term survival of patients with NPC. On the other hand, a retrospective study done in Malaysia reported that the risk of death within five years for NPC patients older than 70 years of age was 3.18 times that of patients younger than 50 years of age [15].

Existing epidemiological data demonstrate that gender is a major factor in the incidence of NPC. The occurrence of NPC in males is 2-3 times that in females; this is similar to the results found in the current study $[16,17]$. Previous studies have reported slightly better, though not significant, long-term survival rates in women with NPC than in men [15]. However, in one Japanese retrospective study, a significantly higher 5-year survival rate was reported for female patients compared to male patients $(\mathrm{P}=0.484)$, through multivariate analysis showed that gender was not an independent prognostic factor for survival in this study [16]. In sharp relations studies done in Macau respectively, suggesting that gender was an independent prognostic factor [17]. Similarly, in the current study, the 5-year OS rate for male and female patients was $43.8 \%$ and $60.3 \%(\mathrm{P}=0.042)$ and the 5-year PFS rate was $39.6 \%$ and $59.3 \%(\mathrm{P}=0.044)$, slightly indicative for gender been also an independent prognostic factor affecting OS and PFS.

Some studies have demonstrated that patients with WHO Type III NPC are more sensitive to radiotherapy, and survive longer, than those with Type I [18]. However, there is not yet enough evidence that supports the idea that patients with different pathological types of NPC require different treatment modalities $[1,19]$. In the current study, patients with WHO Type III NPC accounted for only $6.7 \%$ of cases, while those with Types I and II accounted for only 33.3\% of cases. No statistically significant difference found in OS $(\mathrm{P}=0.088)$ or PFS $(\mathrm{P}=0.368)$ among the different pathological types in the current study, though this is contrary to previously published results $[15,16]$. A retrospective study conducted in Malaysia showed that the risk of death was 1.97 times greater in patients with WHO Types I and II NPC than in patients with type III [15]. Similarly, in a retrospective Japanese study, patients with the non-keratinizing type of NPC (WHO Types III and II) 
were found to have higher 5-year OS and PFS rates than those with the keratinizing type (WHO Type I) [16]. Two studies, conducted in Brazil, and Macao reported results similar to the current study and concluded that there was no significant difference in the 5-year disease-specific survival rates among different histological types of NPC [20,21]. Many studies have confirmed a clear association between long-term survival and NPC clinical stage $[15,16,19,20,21]$. Results of the current study also demonstrated that, as the disease stage increased, the 5-year OS and PFS rates gradually and significantly decreased $\mathrm{P}=0.000$. Multivariate analysis established AJCC staging as an independent prognostic indicator for OS and PFS. Further analysis demonstrated that tumor size (T) and distant metastases (M) are decisive factors for OS and PFS, but that lymph node metastasis $(\mathrm{N})$ staging has no independent prognostic significance for OS or PFS. The 5-year OS and PFS rates in patients with stage T1 were 2.68 and 3.25 times that of patients with T4, respectively. Whiles, in patients with M1, the risk of death was 4.28 times higher, similar to the risk of recurrence and progression 4.30 times higher than in patients with M0.

The efficacy of primary treatment was also found to be an independent prognostic factor affecting long-term survival. Among all 183 patients, the complete remission (CR) rate was $56.5 \%$, progression disease rate $(\mathrm{PD})$ was $14.4 \%$ and the partial remission (PR) rate was $47.1 \%$. The 5-year OS and PFS rates were slightly higher and significant in patients with $\mathrm{CR}$ and $\mathrm{PR}$ than in patients without remission $(\mathrm{P}=0.001$ and $\mathrm{P}=0.000$, respectively). Based on this, the initial treatment modality and its therapeutic efficacy are the major factors affecting the prognosis of patients in all stages of NPC. Measures should be taken to achieve CR or PR during primary treatment as this will improve the long-term survival of patients in all stages of NPC.

NPC is sensitive to radiotherapy and chemotherapy $[22,23]$. Since surgical resection is difficult and the efficacy is poor, the primary treatment for NPC is radiotherapy and chemotherapy is used as an adjuvant option. Surgical resection is limited to cases in which there is a residual tumor or can be used as salvage therapy in cases of local recurrences. Although NPC is relatively sensitive to radiotherapy, the long-term survival for patients with advanced NPC is not ideal [20, 24, $25,34]$. According to the literature, the five-year survival rate for patients with stage IV NPC, who received radiotherapy only, is between $12.5 \%$ and $8.0 \%[25,26]$. Depicting from the study, the appropriate addition of chemotherapy is necessary to improve long-term survival in these patients.

Most studies haveindicated that adding chemotherapy to radiotherapy can improve treatment efficacy and prolong OS in patients with intermediate or advanced NPC, though not all studies have had positive results [9,20, 28-31]. Phua et al. found no difference in the prognosis among patients with different stages of NPC whether chemotherapy was added or not [15]. Also, a retrospective analysis by Chua et al. found that radiotherapy combined with induction chemotherapy resulted in only a mild improvement in PFS and in the relapse rate and no improvement in OS when compared with radiotherapy alone [9]. A phase III clinical trial conducted in patients with locally advanced NPC in China showed that adding adjuvant chemotherapy did not result in improved OS or relapse-free survival when compared with using concurrent chemo-radiotherapy [32]. Chen et al. reported that, in a randomized phase III trial, the 5-year survival in 230 cases of stage II NPC was significantly better in the group treated with combined radiotherapy and chemotherapy compared to the group treated with radiotherapy alone [34]. The prolonged survival in the combined group was mainly attributed to a lower rate of distant metastases; however, restaging these patients according to the latest TNM classification system [2014] revealed that a considerable portion of the patients should have been categorized as stage III [11].

The group (s) that received combined chemotherapy and radiotherapy was compared with the group that received radiotherapy only to determine the effect of adding chemotherapy on patient survival in our study. The 5-year OS and PFS rates in the combined chemotherapy and radiotherapy groups (CCRT+RT+AC, NAC+RT+CCRT) were significantly higher than in the radiotherapy only group ( $\mathrm{P}=0.012$ and $\mathrm{P}=0.005$, respectively). Also, according to AJCC stage-based subgroup analysis, there was no much difference between the three groups in the 5-year OS rate of patients with stages I, II and III NPC, although the 5-year OS rate of patients with stage IV NPC was significantly higher in the chemotherapy combined with radiotherapy groups $(\mathrm{CCRT}+\mathrm{RT}+\mathrm{AC}, \mathrm{NAC}+\mathrm{RT}+\mathrm{CCRT})$ than in the radiotherapy only group. For patients with stage I or II NPC, the 5-year PFS rate was not significantly different in the combined groups vs. the radiotherapy only group $(\mathrm{P}=0.550)$. Conversely, for patients with stage III or IV NPC, the rate of 5-year PFS was significantly higher in the combined groups (CCRT $+\mathrm{RT}+\mathrm{AC}$, 
$\mathrm{NAC}+\mathrm{RT}+\mathrm{CCRT}$ ) than in the radiotherapy only group $(\mathrm{P}=0.025$ and $\mathrm{P}=0.008$, respectively $)$.

Patients with stages I or II NPC will likely not benefit from the addition of chemotherapy, in terms of long-term survival and PFS. However, for patients with stage III NPC, adding chemotherapy can improve PFS to a certain degree though it may not improve OS and in patients with stage IV NPC, the addition of chemotherapy can significantly prolong both OS and PFS. A random trial from endemic regions of China also showed the addition of concurrent and adjuvant chemotherapy to RT provides survival benefits to patients with stage III through IVB NPC [31].

\section{Conclusions}

The current study indicates that in clinical practice, it is recommended that chemotherapy be added to radiotherapy for patients with stage IV NPC. Treatment modalities may include induction chemotherapy, concurrent chemoradiotherapy, and adjuvant or palliative chemotherapy treatment after radiotherapy. In patients with stage III NPC, the treatment should be based on the individual. Chemotherapy may be considered for patients that are otherwise in good general health or for patients that have a relatively advanced stage of NPC. In patients with mid and early stages of NPC, such as stage II or lower, chemotherapy is not really recommended.

The study conducted using conventional radiotherapy during the period under review will also serve as the basis for future study in IMRT survival assessments.

The study used was a descriptive cross-sectional, retrospective analysis and the clinical stage between the three groups. Sample method used was Universal sampling. This will invariably reduce bias from the study. However, a cohort study or randomized phase III could also be used if necessary for future study.

\section{Declarations and Acknowledgements}

\section{Authors' contributions}

Clement Arthur analyzed the data and wrote the paper. Hao Ruan, Collins Koranteng Osei, and Akparibila Joseph Azure and Xun Bi prepared and calculated the data.
Xiaofeng Wang, Xue Jun Zhou, Sha Liu, and Ping Zhou, data check, analysis, and information retrieval. Clement Arthur and ZhongLin $\mathrm{Mu}$ conceived of the study and participated in its design, Technical advice, and coordination. All authors read and approved the final manuscript.

\section{Acknowledgments}

We acknowledge the co-operation of all the population-based cancer registries in providing cancer statistics, data collection, sorting, and verification, and database creation. We thank StaffOtorhinolaryngology Department of Radiotherapy, the Department of Haemato-oncology, Hainan Medical College First Affiliated Hospital, the ethical committee, the research department, the statistics department of Hainan Medical College and all the above-listed authors for their help in the facilitation of the study.

Support By: National Natural Science Foundation of China (NO: 81660171)

\section{Competing interests}

All authors declare that there are no conflicts of interest.Support By: National Natural Science Foundation of China (NO: 81660171)

\section{Course Title: ENT}




\section{References}

1. Sun LM, Li CI, Huang EY, et al. (2007) Survival differences by race in nasopharyngeal carcinoma. Am J Epidemiology 165:271-278.

2. Marks JE, Philips JL, Menck HR (1998) The National Cancer Data Base report on the relationship of race and national origin to the histology of nasopharyngeal carcinoma. Cancer 83:582-588.

3. Nelson V, Rademaker A, Kaklamani V (2013) Paradigm of polyendocrine therapy in endocrine-responsive breast cancer: the role of fulvestrant. Chin ClinOncol10: 2304-3865.

4. Lin A, Swisher-McClure S, Millar LB, et al. (2012) Proton therapy for head and neck cancer: current applications and future directions. Transl Cancer Res 1:255-263.

5. Li X, Xiao S, Li Y, et al. (2013) Clinical anti-angiogenic effect of recombinant adenovirus-p53 combined with hyperthermia for advanced cancer. Chin J Cancer Res 25:749755.

6. Chedid HM, Franzi SA, Dedivitis RA, et al. (2008) Assessment of clinical and therapeutic factors in patients with nasopharyngeal undifferentiated carcinoma. Braz J Otorhinolaryngol 74:566-570.

7. Chua DT, Ma J, Sham JS, et al. (2006) Improvement of survival after addition of induction chemotherapy to radiotherapy in patients with early-stage nasopharyngeal carcinoma: Subgroup analysis of two Phase III trials. Int J Radiat Oncol Biol Phys 65:1300-1306.

8. Cheng SH, Tsai SY, Yen KL, et al. (2000) Concomitant radiotherapy and chemotherapy for early-stage nasopharyngeal carcinoma. J Clin Oncol 18:2040-2045.

9. Chua DT, Ma J, Sham JS, et al. (2005) Long-term survival after cisplatin-based induction chemotherapy and radiotherapy for nasopharyngeal carcinoma: a pooled data analysis of two phases III trials. J ClinOncol23:1118-1124.

10. MB, Edge SB, Greene FL, et al. (2017) eds. AJCC Cancer Staging Manual. 8th ed. New York: Springer 67:93-99.

11. Barnes L, Eveson JW, Reichart P, et al. (2005) eds. World Health Organization classification of tumors. Pathology and genetics of head and neck tumors. Lyon: IARC Press 85:74.

12. Zhang W, Dou H, Lam C, et al. (2013) Concurrent chemoradiotherapy with or without adjuvant chemotherapy in intermediate and locoregionally advanced nasopharyngeal carcinoma. Tumour Biol 34:1729-1736.
13. Ma J, Mai HQ, Hong MH, et al. (2001) Results of a prospective randomized trial comparing neoadjuvant chemotherapy plus radiotherapy with radiotherapy alone in patients with locoregionally advanced nasopharyngeal carcinoma. J Clin Oncol 19:1350-1357.

14. Miller AB, Hoogstraten B, Staquet M, et al. (1981) reporting results of cancer treatment. Cancer 47:207-214.

15. Phua CE, Tan BS, Yong TK, et al. (2011) Retrospective analysis of results of treatment for nasopharyngeal carcinoma in Penang General Hospital from 2001-2005. Asian Pac J Cancer Prev 12:3197-3200.

16. Kawashima M, Fuwa N, Myojin N, et al. (2004) A multi-institutional survey of the effectiveness of chemotherapy combined with radiotherapy for patients with nasopharyngeal carcinoma. Jpn J Clin Oncol 34:569-583.

17. Hui EP, Leung SF, Au JS, et al. (2004) Lung metastasis alone in nasopharyngeal carcinoma: a relatively favorable prognostic group. A study by the Hong Kong Nasopharyngeal Carcinoma Study Group. Cancer 101:300-306.

18. Goto Y, Kodaira T, Fuwa N, et al. (2013) Alternating chemoradiotherapy in patients with nasopharyngeal cancer: prognostic factors and proposal for individualization of therapy. J Radiat Res 54:98-107.

19. Geara FB, Sanguineti G, Tucker SL, et al. (1997) Carcinoma of the nasopharynx treated by radiotherapy alone: determinants of distant metastasis and survival. Radio Ther Oncol 43:53-61.

20. Farias TP, Dias FL, Lima RA, et al. (2003) Prognostic factors and outcome for nasopharyngeal carcinoma. Arch Otolaryngol Head Neck Surg 129:794-799.

21. Lee AW, Sze WM, Au JS, et al. (2005) Treatment results for nasopharyngeal carcinoma in the modern era: the Hong Kong experience. Int J Radiat Oncol Biol Phys 61:11071116.

22. al-Sarraf M, McLaughlin PW. (1995) Nasopharyngeal carcinoma: choice of treatment. Int J Radiat Oncol Biol Phys 33:761-763.

23. Schantz SP, Harrison LB, Forastiere AA (1997) Tumors of the nasal cavity and paranasal sinuses, nasopharynx, oral cavity, and oropharynx. In: DeVita VT, Hellman S, Rosenberg SA. eds. Cancer: Principles and Practice of Oncology. 5th ed. Philadelphia, Pa: Lippincott-Raven 741-801.

24. Teo P, Yu P, Lee WY, et al. (1996) Significant prognosticators after primary radiotherapy in 903 nondisseminated nasopharyngeal carcinoma evaluated by computed tomography. Int J Radiat Oncol Biol Phys 36:291-304. 
25. Lee AW, Poon YF, Foo W, et al. (1992) Retrospective analysis of 5037 patients with nasopharyngeal carcinoma treated during 1976-1985: overall survival and patterns of failure. Int J Radiat Oncol Biol Phys 23:261-270.

26. Heng DM, Wee J, Fong KW, et al. (1999) Prognostic factors in 677 patients in Singapore with non disseminated nasopharyngeal carcinoma. Cancer 86 1912-1920.

27. Marcial VA, Hanley JA, Chang C, et al. (1980) Splitcourse radiation therapy of carcinoma of the nasopharynx: results of a national collaborative clinical trial of the Radiation Therapy Oncology Group. Int J Radiat Oncol Biol Phys 6:409414.

28. Park KH, Kim JS, Park Y, et al. (2010) Concurrent chemoradiation followed by adjuvant chemotherapy in patients with locoregionally advanced nasopharyngeal carcinoma in Korea. Cancer Chemother Pharmacol 66:643-651.

29. Chan AT, Teo PM, Ngan RK, et al. (2002) Concurrent chemotherapy-radiotherapy compared with Radiotherapy alone in locoregionally advanced nasopharyngeal carcinoma: progression-free survival analysis of phase III randomized trial. J Clin Oncol 20:2038-2044.

30. Lee AW, Tung SY, Chua DT, et al. (2010) Randomized trial of radiotherapy plus concurrent-adjuvant chemotherapy vs radiotherapy alone for regionally advanced nasopharyngeal carcinoma. J Natl Cancer Inst 102:1188-1198.

31. Chen Y, Sun Y, Liang SB, et al. (2013) Progress report of a randomized trial comparing long-term survival and late toxicity of concurrent chemoradiotherapy with adjuvant chemotherapy versus radiotherapy alone in patients with stage III to IVB nasopharyngeal carcinoma from endemic regions of China. Cancer119:2230-2238.

32. Chen L, Hu CS, Chen XZ, et al. (2012) Concurrent chemoradiotherapy plus adjuvant chemotherapy versus concurrent chemoradiotherapy alone in patients with locoregionally advanced nasopharyngeal carcinoma: a phase 3 multicentre randomized controlled trial. Lancet Oncol 13:163-171.

33. Chen QY, Wen YF, Guo L, et al. (2011) Concurrent chemoradiotherapy vs radiotherapy alone in stage II nasopharyngeal carcinoma: phase III randomized trial. J Natl Cancer Inst 103:1761-1770.

34. J. Daoud, N. Toumi, W. Siala, A. Ghorbel, M. M. Drira, et al. (2007) "Results of a prospective randomized trial comparing conventional radiotherapy to split course fractionated radiation therapy in patients with nasopharyngeal carcinoma," Radiotherapy and Oncology 85:17-23.
Submit your manuscript to a JScholar journal and benefit from:

๑ Convenient online submission

ฯ Rigorous peer review

- Immediate publication on acceptance

ๆ Open access: articles freely available online

ब High visibility within the field

ब Better discount for your subsequent articles

Submit your manuscript at http://www.jscholaronline.org/submit-manuscript.php 